\title{
512 PHASE I DOSE ESCALATION OF KD033, A PDL1-IL15 BISPECIFIC MOLECULE, IN METASTATIC AND ADVANCED SOLID TUMORS
}

${ }^{1} J a s o n$ Luke*, ${ }^{2}$ Anthony Olszanski, ${ }^{3}$ Igor Puzanov, ${ }^{3}$ Christos Fountzilas, ${ }^{4}$ Lee Rosen, ${ }^{5}$ Dan Lu, ${ }^{5}$ Adrian Hackett, ${ }^{5}$ Stella Martomo, ${ }^{5}$ Olivier Schueller, ${ }^{5}$ David Eiznhamer, ${ }^{5}$ Alessandro Mora, ${ }^{5}$ Miranda Ross, ${ }^{5}$ Jeegar Patel. ${ }^{1}$ UPMC, Pittsburgh, PA, USA; ${ }^{2}$ Fox Chase Cancer Center, Philadelphia, PA, USA; ${ }^{3}$ Roswell Park Cancer Center, Buffalo, NY, USA; ${ }^{4}$ UCLA Division of Hematology-Oncology, Los Angeles, CA, USA; ${ }^{5}$ Kadmon Corporation, NEW YORK, NY, USA

Background While IL-2 and IL-15 signal through the shared IL-2/15 $\beta \gamma$ receptor, IL-15 does not directly expand regulatory $\mathrm{T}$ cells (Tregs)or mediate activation-induced cell death and may have an improved therapeutic index. KD033 is a fusion antibody combining a fully human, high affinity anti-human Programmed Death Ligand 1 (PD-L1) IgG1 antibody with the human IL-15 receptor alpha (IL15R $\alpha$ ) sushi domain and human IL-15 (IL-15). KD033 and its mouse cross-reactive surrogate molecule, srKD033, have been extensively characterized in multiple in vitro and in vivo nonclinical studies and have demonstrated robust efficacy and therapeutic benefits compared to IL-15 alone.

Methods This is a phase 1, open-label, multiple ascending dose, multi-center clinical trial being conducted in patients with metastatic or locally advanced solid tumors (NCT04242147). The primary objective is to determine the safety, tolerability, and MTD of KD033. Secondary objectives include characterization of $\mathrm{PK}$ and immunogenicity, evaluation of CD8 $+\mathrm{T}$ and NK cell activation, and assessment of best overall response and duration of response. KD033 is administered by IV infusion over 30 minutes every 14 days. The study design follows $3+3$ escalation investigating dose ranges from $3 \mu \mathrm{g} / \mathrm{kg}$ to $600 \mu \mathrm{g} / \mathrm{kg}$.

Results A total of 12 patients have received treatment. Three patients were dosed in Cohort 1 (C1), four patients were dosed in Cohort 2 (C2), and three patients were dosed in Cohort 3 (C3). Two patients in Cohort 4 (C4) have been dosed. Through three dose escalation cohorts $(3 \mu \mathrm{g} / \mathrm{kg}-50$ $\mu \mathrm{g} / \mathrm{kg}$ ), no dose-limiting toxicities have been reported. Grade 1-2 treatment-related toxicities resolved within 24 hours with supportive management. Grade 4 decreases in lymphocytes were noted the day after dosing in C3 and C4, which resolved on day 3 and were expected. One patient (adenoid cystic carcinoma) in C1 was shown to have stable disease for more than 6 months and one patient (metastatic gastric adenocarcinoma) in C3 was shown to have stable disease for more than 4 months.

Conclusions To date, KD033 has been well tolerated in all subjects with on-mechanism pharmacodynamics consistent with IL-15 agonism.

Ethics Approval This study obtained ethics approval from WIRB.

http://dx.doi.org/10.1136/jitc-2021-SITC2021.512 